\title{
Allen key completely in male urethra: a case report Michael Mitterberger ${ }^{1 *}$, Reinhard Peschel ${ }^{1}$, Ferdinand Frauscher ${ }^{2}$ and Germar M Pinggera ${ }^{1}$
}

\author{
Addresses: ${ }^{1}$ Department of Urology, Medical University Innsbruck, Anichstrasse 35, A-6020 Innsbruck, Austria \\ ${ }^{2}$ Department of Radiology, Medical University Innsbruck, Anichstrasse 35, A-6020 Innsbruck, Austria \\ Email: MM* - michael.mitterberger@i-med.ac.at; RP - reinhard.peschel@uki.at; FF - ferdinand.frauscher@i-med.ac.at; \\ GMP - germar.pinggera@uki.at \\ * Corresponding author
}

Received: 23 April 2009 Accepted: 18 June 2009 Published: 20 July 2009

Cases Journal 2009, 2:7408 doi: 10.4076/1757-1626-2-7408

This article is available from: http://casesjournal.com/casesjournal/article/view/7408

(C) 2009 Mitterberger et al; licensee Cases Network Ltd.

This is an Open Access article distributed under the terms of the Creative Commons Attribution License (http://creativecommons.org/licenses/by/3.0), which permits unrestricted use, distribution, and reproduction in any medium, provided the original work is properly cited.

\begin{abstract}
Introduction: Various cases of self-inflicted foreign bodies in the male urethra have been reported. Most of them are associated with autoerotic stimulation, psychiatric disorders or intoxication.

Case presentation: We report the first case of a patient who put an Allen key completely in his urethra. The patient presented with dysuria, haematuria and penile pain.

Conclusion: A self-inflicted urethral foreign body is a rare situation. Endoscopic removal is the recommended first-line treatment and if unsuccessful, open procedures may be necessary.
\end{abstract}

\section{Introduction}

Various cases of self-inflicted foreign bodies in the male urethra have been reported [1]. Most of them are associated with autoerotic stimulation, psychiatric disorders or intoxication [2]. The patients present with dysuria, haematuria, urinary retention, penile pain or swelling [3]. We report about a patient who put an Allen key completely in his urethra.

\section{Case presentation}

A 51-year-old Caucasian male presented to the emergency room and stated that he has an Allen key in his urethra since two days. The patient could void and had bloody urethral discharge. Further he had made several unsuccessful attempts to remove it. His physical examination showed a normal urethral meatus and a palpable foreign body within the penis. An x-ray of pelvis demonstrated a $9 \mathrm{~cm}$ long metallic object in the anterior urethra, corresponding an Allen key (Figures 1 and 2). The patient was not married and his socioeconomic status was of upper class. It was the first time he had ever self-inflicted a foreign body in his urethra and he had no history of drug addiction or psychiatric illness. After giving his formal consent, the patient was taken to the operating room. Under general anesthesia and fluoroscopic control, an attempt was made to pull out the foreign body with a $22 \mathrm{Fr}$ cystoscope. This was unsuccessful, due to lack of space. Then an external urethrotomy of the anterior urethra was performed, the Allen key was removed and an $18 \mathrm{~F}$ urethral catheter was placed (Figures 3 and 4). The patient was discharged two days later with the catheter. After three weeks under oral antibiotic therapy a voiding 


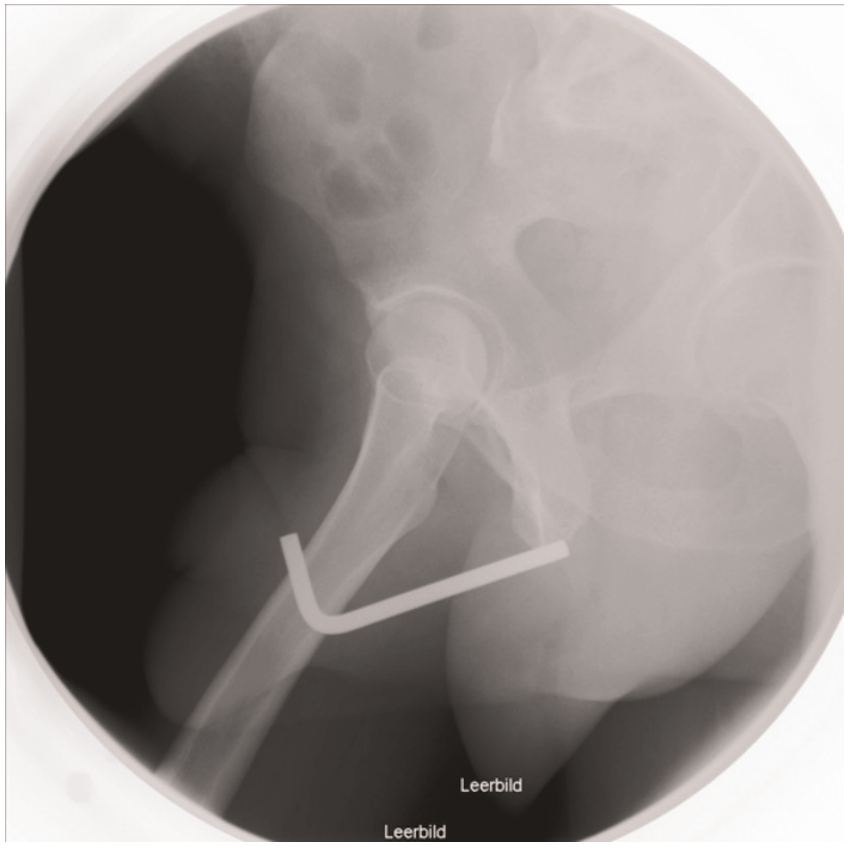

Figure I. X-ray of the pelvis showing the Allen key completely in the anterior urethra.

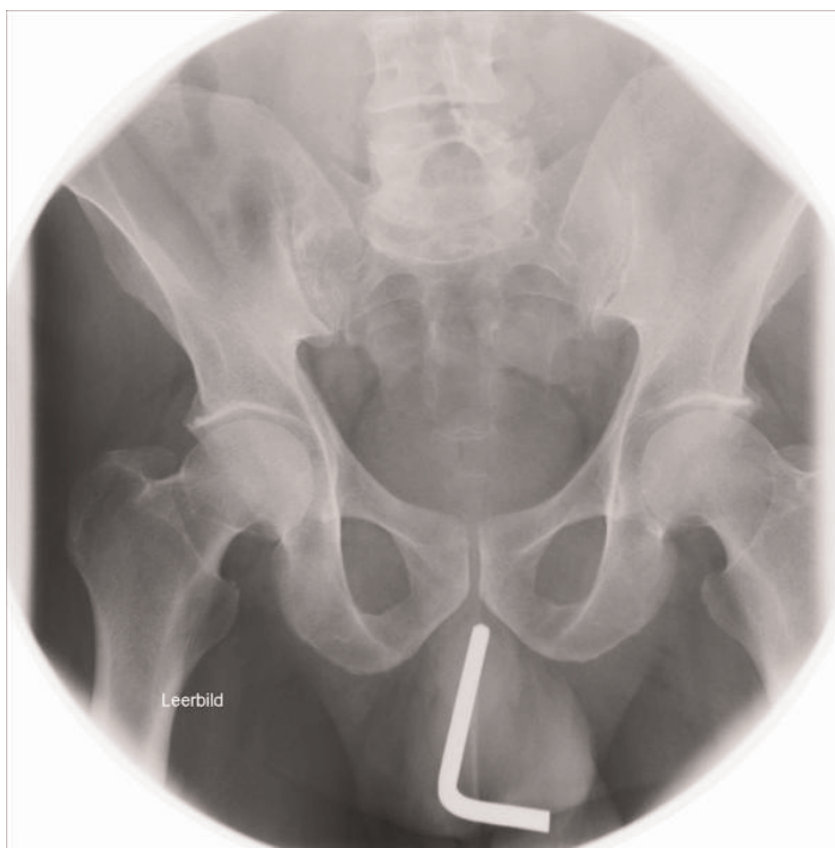

Figure 2. X-ray of the pelvis showing the Allen key in the urethra.

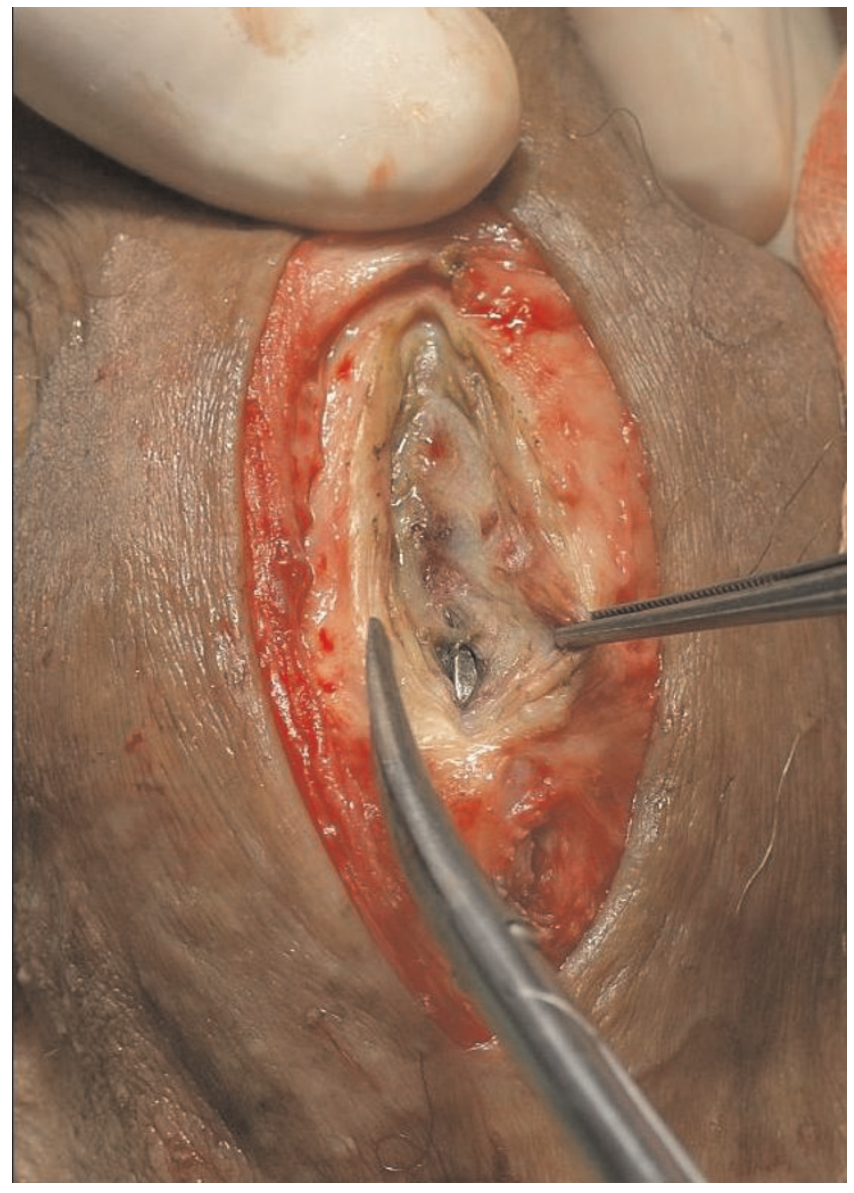

Figure 3. External urethrotomy of the anterior urethra showing the metallic object in the urethra.

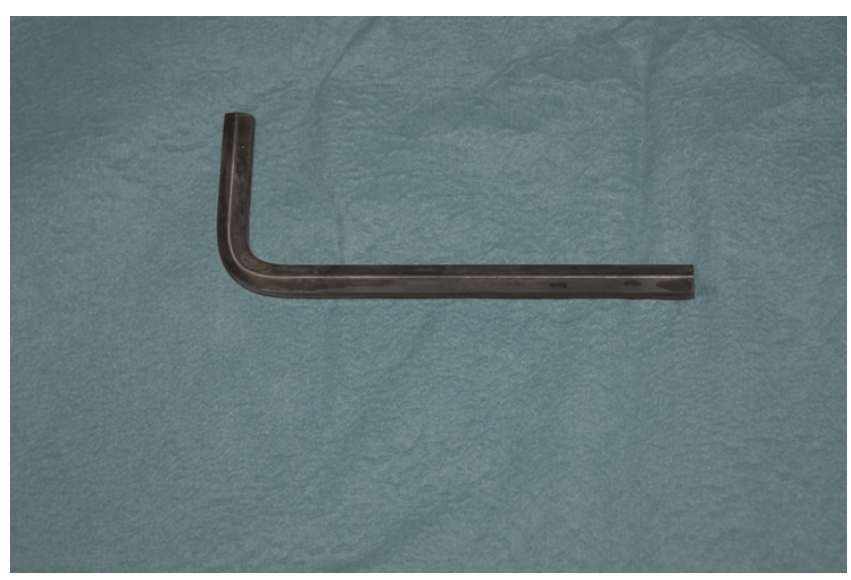

Figure 4. Allen key removed from urethra. 
cystourethrogram (which detected no abnormalities) was performed and the patient was discharged again. Weeks later the patient presented himself again showing a long penile stricture. Therefore a urethral reconstruction had to be performed.

\section{Discussion}

Various self-inflicted foreign bodies have been reported in the male urethra [1]. Objects like nuts, pens, wire and others have been described. The clinical presentation of the patient may vary from asymptomatic to swelling of external genitalia, dysuria, poor urinary stream, urethral discharge or urinary tract infection [3]. The main reason for foreign body self-insertion is of sexual nature, like masturbation or sexual gratification [2]. Also a mental illness or drug intoxication may be the reason. Most of the patients feel guilty and ashamed and do not seek medical help until they experience severe voiding problems. In our case, the patient underwent several unsuccessful attempts to remove the foreign body and came with a delay of two days.

Interesting underlying psychiatric and psychoanalytic theories have been postulated [4]. However, the psychiatric evaluation is controversial as many of these patients are psychologically normal. Also in our case, the psychiatrist evaluation was normal and the patient revealed no signs of depression or impulsive behavior.

The primary goal of the treatment should be removal of the foreign body with little damage to the urethra as possible. Further various methods of foreign body removal have been described, depending on the form, size and mobility of the body and its location in the urethra [5]. Endoscopic therapy is the standard care, but in cases where the foreign body cannot be removed open surgery becomes necessary. In our case, due to the ninety degree angle of the Allen key, endoscopic removal was unsuccessful and an external urethrotomy had to be performed.

Known long term complications of self-inflicted urethral foreign bodies are urethral stricture or diverticulum, incontinence or erectile dysfunction. Further the rationale for the behaviour should be investigated to prevent recurrence [1].

\section{Conclusion}

A self-inflicted urethral foreign body is a rare situation. Endoscopic removal is the recommended first-line treatment and if unsuccessful, open procedures may be necessary.

\section{Consent}

Written informed consent was obtained from the patient for publication of this case report and accompanying images. A copy of the written consent is available for review by the journal's Editor-in-Chief.

\section{Competing interests}

The authors declare that they have no competing interests.

\section{Authors' contributions}

MM wrote the case report. RP and GP provided clinical care of the patient during his treatment. FF provided the images. GP supervised the writing of the case report and suggested amendments. All authors read and approved the final manuscript.

\section{References}

I. Rahman NU, Elliott SP, McAninch JW: Self-inflicted male urethral foreign body insertion: endoscopic management and complications. BJU Int 2004, 94: I05I-I 053.

2. Stravodimos KG, Koritsiadis G, Koutalellis G: Electrical wire as a foreign body in a male urethra: a case report. J Med Case Reports 2009, 3:49.

3. Sukkarieh T, Smaldone M, Shah B: Multiple foreign bodies in the anterior and posterior urethra. Int Braz I Urol 2004, 30:2 19-220.

4. Kim ED, Moty A, Wilson DD, Zeagler D: Treatment of a complete lower urinary tract obstruction secondary to an expandable foam sealant. Urology 2002, 60:164.

5. van Ophoven A, deKernion JB: Clinical management of foreign bodies of the genitourinary tract. J Urol 2000, 164:274-287.

\section{Do you have a case to share?}

\section{Submit your case report today}

- Rapid peer review

- Fast publication

- PubMed indexing

- Inclusion in Cases Database

\section{Any patient, any case, can teach us something}

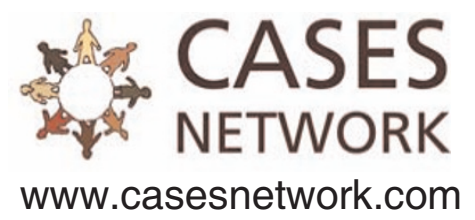

